\title{
Archiv und Politik
}

\section{Zu Stifter-Handschriften in Prag, Linz, München und Genf}

Flair und Faszination von Bibliotheken - und hier in Sonderheit ihrer Lesesäle als Kernzonen der Vergegenwärtigung des Vergangenen - sind in ihrer ganz und gar unvergleichlichen Atmosphäre vielfach beschrieben und beschworen worden (vgl. hierzu stellvertretend Jammers et al. 2002; Rossner 2016): als Orte stillen Dialogs in intensiver Zuwendung und kontemplativer Achtsamkeit wie ebenso auch durchaus wacher sozialer Interaktion (vgl. Schley 2008). Dies gilt in besonderem Maße auch für deren ,Schatzkammern', also die höchst eigentümliche Aura, die nicht nur von einer Handschrift ausgeht, sondern auch in den zu ihrem Studium bereitgestellten, in der Regel nochmals exklusiveren Räumlichkeiten ,buchstäblich“ fühlbar wird. Mir jedenfalls scheinen sie in dem ihnen innewohnenden Fluidum von konzentrierter Hingabe und unabgelenkter Versenkung, von offensichtlicher Demut und tiefem Respekt vor den Zeugnissen unserer Geschichte durchaus als säkulare Brüder der Gotteshäuser, dies im Bewusstsein, dass viele der in diesen Bergwerken der Erinnerung beschäftigten Archivarinnen und Archivare diese Eindrücke womöglich kaum teilen oder gar belächeln mögen. Wer jedoch - damals noch als Doktorand - in Marbach einmal vor dem leibhaftigen Stapel jener Papiere stehen durfte, die auf der ersten Seite mit den Worten „Jemand mußte Josef $\mathrm{K}$. verläumdet haben ..." einsetzen, wird dies als einen der feierlichsten Momente seines literaturwissenschaftlichen Lebens in Erinnerung behalten. ${ }^{1}$ Womit wir, zumindest was den Autor dieses Satzes betrifft, an einem der Orte angelangt sind, zu denen dieser Beitrag führen wird.

„Über ein literarisches Archiv zu sprechen ist sicher keine kurzweilige Angelegenheit. Äußerlich bietet sich dem Besucher zumeist ein Bild verstaubter Regale, gefüllt mit unansehnlichen Schachteln“ (Hofman 1984, 109). Möglicherweise liegt vielen dieses Bild, wie es der Prager Germanist Alois Hofman zeichnete, ja näher als das Pathos der vorangegangenen Eingangsworte, wenngleich auch er unmittelbar anschließend demgegenüber ebenso die „Ausstrahlung“ und das „Fluidum“ der in ihnen aufbewahrten Dokumente, und hier insbeson-

1 Bewusst wurde hier der persönliche Ton des Vortrags beibehalten, der es erlaubt, den VeranstalterInnen für die Einladung zum Internationalen Symposium Logiken der Sammlung. Das Archiv zwischen Strategie und Eigendynamik zu danken, wo dieser für den Druck überarbeitete Beitrag am 25. April 2019 im Adalbert-Stifter-Institut in Linz erstmals öffentlich vorgetragen wurde. 
dere „der persönlichen Handschrift“ (Hofman 1984, 109) eines Dichters hervorhebt. Die zitierten Passagen finden sich in einem 1984 in Linz gehaltenen Vortrag mit dem Titel Bericht über das Prager Adalbert-Stifter-Archiv. Was nicht nur eine erste Ortsbesichtigung erlaubt, sondern zugleich mitten in die spezifische Fragestellung - nämlich dem Zusammenhang von ,Archiv und Politik - führt.

Dass auch das scheinbar weltabgewandte, oft als selbstvergessenes Glasperlenspiel getadelte historisch-kritische Edieren, die tatsächlich oft mühsame Entzifferung handschriftlicher Vorlagen und ihre Übertragung in Lesetext und Apparat, keine Beschäftigung ist, die sich im luftleeren, sprich: politisch unberührten Raum ereignet, bedarf unter Fachleuten wohl keiner näheren Erläuterung, obgleich die erste Assoziation hier eher wissenschaftspolitischen, noch genauer: ökonomischen Aspekten gelten dürfte - etwa dem Zugang zu Fördermitteln öffentlicher wie privater Art, worauf solche Langzeit-Projekte zu ihrer Sicherung oft über Jahrzehnte hinweg angewiesen sind und deshalb unter einem nicht unbeträchtlichen Erfolgs- wie Rechtfertigungsdruck stehen.

Im Falle Adalbert Stifters - 1805 im böhmischen Oberplan geboren und 1868 in Linz verstorben - sind die beiden historisch-kritischen Werkausgaben, die mit den Sammlungen seines handschriftlichen Nachlasses jeweils in ursächlichem Zusammenhang stehen, jedoch in Planung wie Projektverlauf von den unmittelbaren zeitpolitischen Umständen nicht zu trennen. Dies betrifft im ersten Teil das Prag an der Wende zum 20. Jahrhundert und die dort von August Sauer begründete Prag-Reichenberger-Ausgabe (im Folgenden PRA), sodann die noch nicht abgeschlossene, immerhin aber bereits 39 Bände umfassende Historisch-Kritische Ausgabe der Werke und Briefe Adalbert Stifters (im Folgenden HKG), deren Vorgeschichte unmittelbar in den kulturpolitischen Konstellationen der 60er-Jahre des vergangenen Jahrhunderts wurzelt. $\mathrm{Zu}$ Linz und München sowie Genf dann im zweiten Teil.

Die Geschichte der PRA, in 25 Bänden erschienen und mit dem letzten, nicht unbedingt geglückten Band erst 1979 abgeschlossen (vgl. zu diesem Bergner 1982), ist gut dokumentiert und hier nicht das Thema, deshalb dazu in aller Kürze (vgl. allgemein zu den beiden historisch-kritischen Stifter-Werkausgaben: Stüben 2017; Hettche und John 2017). Als der Prager Ordinarius August Sauer den Plan hierzu erstmals 1898 vorlegte, war die dortige Karls-Universität bekanntlich seit dem 28. Februar 1882 per Gesetz in eine deutsche und tschechische, jeweils selbständige Alma Mater getrennt worden, was auch ihre germanistischen Seminare betraf (vgl. Höhne 2017, 18-21). In jener Periode sich verschärfender, zunehmend unversöhnlicher nationaler Konflikte und ethnischer Emanzipationsbestrebungen, wie sie die Spätphase der Habsburger Monarchie generell kennzeichneten, war schon die Gründung der „Gesellschaft zur Förderung deutscher Wissenschaft, Kunst und Literatur in Böhmen“ im Jahre 1890 ein bewusster Akt nationaler 
Identitätsstiftung gewesen, ebenso die von Sauer 1893 ins Leben gerufene Bibliothek deutscher Schriftsteller in Böhmen. Im März und April 1899 schließlich beschloss die „Gesellschaft“ die Neuedition einer Werkausgabe Stifters, die den damals ,modernen' textkritischen Ansprüchen genügen sollte; das Modell hierfür bildete die in jenen Jahrzehnten entstehende Weimarer ,Sophien-Ausgabe‘ der Werke Goethes, wobei - sicherlich anders als heute, aber nicht unwichtig zu erwähnen - in der neugermanistischen Philologie die unter Beweis gestellte Vertrautheit mit „Archiv- und Editionsarbeit“ (Riener 2011, 289) einen wesentlichen Prüfstein für die künftige akademische Karriere bildete. Dass, mentalitätsgeschichtlich am Vorbild Goethe orientiert, Stifter damit durchaus vergleichbar als repräsentativer deutsch-böhmischer ,Klassiker‘ inthronisiert werden sollte, machen die beiden 1901 und 1906 veröffentlichten Berichte Sauers zur Edition ebenso deutlich wie sein Vorwort zum ersten 1904 erschienenen Band der Ausgabe (Stifter, PRA, Bd. 1, VII-XXX), ${ }^{2}$ die dann - von den Wirren des Ersten Weltkriegs eben so wenig verschont wie von den institutionellen Umstrukturierungen infolge der Gründung der tschechoslowakischen Republik - seit 1927 in Reichenberg, dem heutigen Liberec, verlegt wurde. Das hier implementierte harmonisierende Bild Stifters als regionalem Böhmerwalddichter fernab aller Traditionsbrüche der literarischen Moderne um 1900 sollte sich rezeptionsgeschichtlich nicht nur hartnäckig bis weit in die zweite Jahrhunderthälfte behaupten, sondern, wie zu zeigen sein wird, auch unmittelbar auf dessen Archivierung auswirken.

In unserem Zusammenhang ist dabei der am 3. März 1902 von der „Gesellschaft zur Förderung deutscher Wissenschaft“ beschlossene öffentliche Aufruf relevant, mit Schenkungen, zeitweiligen Überlassungen oder aber Verkäufen zur Gründung und dem Aufbau eines „Stifter-Archivs“ beizutragen. Waren in den Jahren nach Stifters Tod im Januar 1868 weite Teile des Nachlasses in den Besitz seines in Pest, später in Preßburg ansässigen Verlegers Gustav Heckenast (18111878) übergegangen, bleibt allenfalls partiell rekonstruierbar, was Stifters Witwe Amalia (1811-1883) Besuchern wie brieflichen Bittstellern bereitwillig an Autographen überließ. Dank intensiver Sammlertätigkeit wie vor allem auch großzügiger Schenkungen, etwa durch den Wiener Industriellen und Kunstmäzen Karl Adolf Ludwig Freiherr Bachofen von Echt (1830-1922), verfügte das Prager StifterArchiv so nicht nur über Handschriften von Stifters frühestem Prosafragment Julius wie der späten Erzählungen Nachkommenschaften, Der Kuß von Sentze und

2 In der „Einführung“ zu den Studien. Erster Band heißt es gleich eingangs: „Unter den Dichtern, die Böhmens deutsche Erde hervorgebracht hat, nimmt Adalbert Stifter unbestritten die erste Stelle ein. Er allein unter ihnen hat sich einen festen Platz in der Weltliteratur errungen“ (Stifter, PRA 1, VII). 
Der fromme Spruch, sondern vor allem über einen Fundus von mehr als 700 Briefen von und rund 1.000 Briefen an Stifter; das Kernstück dieses Konvoluts bilden die 284 Briefe an seinen Verleger und Gönner Gustav Heckenast, wobei dessen Korrespondenz mit Stifter bis heute leider als verschollen gelten muss. Spannend, falls dieses Adjektiv angesichts der Katastrophengeschichte des 20. Jahrhunderts überhaupt angemessen ist, in jedem Fall aber nicht leicht zu ermitteln, war das weitere Schicksal dieses Archivs in der ersten Jahrhunderthälfte, das - so die Recherchen - stets in Prag verblieb, wenngleich unter nomenklatorisch jeweils veränderter Obhut. 1924 wurde die „Gesellschaft zur Förderung ...“ in „Deutsche Gesellschaft für Wissenschaft und Künste für die Tschechoslowakische Republik" umbenannt, die nach der 1938 erfolgten Okkupation durch deutsche Truppen 1941 in eine „Deutsche Akademie der Wissenschaften in Prag“ umgewandelt wurde (vgl. Hoskovec 2010, 30). Nach Ende des Kriegs schließlich gingen die Bestände in die Obhut der „Národní knihovna“ (Nationalbibliothek) über, wo sich das Stifter-Archiv in deren Handschriftensammlung im Klementinum unweit der Karlsbrücke bis heute befindet.

Wo es allerdings - trotz weiterer wissenschaftlicher Frequentierung - für die scientific community zumindest bis 1962 in einen Dornröschenschlaf fiel, was nicht nur - politics again - der relativen Undurchlässigkeit des ,Eisernen Vorhangs' geschuldet war. Erst 1962 nämlich veröffentlichte der bereits erwähnte, an der Prager Akademie der Wissenschaften tätige Alois Hofman in der Vierteljahrsschrift des Adalbert-Stifter-Institutes auf 170 Seiten eine detaillierte Inventarliste, die - von einem frühen Bericht Sauers abgesehen - erstmals über die in Prag archivierten Stifteriana informierte (vgl. Hofman 1962; dazu auch Tvrdík 1995, 253). Sie bildet bis heute die einzige Informationsquelle, wobei ,Stifteriana‘ hier buchstäblich zu verstehen ist. Die mehrere hundert Seiten umfassenden Abschriften der Briefe Stifters etwa, die sein Nachlassverwalter Johann Aprent (1823-1893) für die Herausgabe seiner dreibändigen Briefausgabe (Aprent 1869) angefertigt hat, sind unter der Signatur „15“ zwar archiviert, aus Hofmans Übersicht aber nur indirekt zu entschlüsseln, was editionsphilologisch insofern von großer Bedeutung ist, da Aprent diese Briefe für seine Auswahl nicht nur bearbeitet, sondern einige Dokumente wegen ihres ,unmännlichen Charakters` eingestandenermaßen auch vernichtet hat, so dass für diese Verluste seine Abschriften die nunmehr einzigen Quellen bilden (vgl. Doppler 2007). Nun sind Kürzungen und Auslassungen mit Rücksicht auf die Privatsphäre auch heute in zeitgenössischen Briefeditionen durchaus üblich, wie rabiat, rigoros und bedenklich jedoch Aprents Streichungen nicht selten sind, mag jenes Beispiel vor Augen führen, wo Stifters ambivalente Passage „Die Kunst kann Ihr Tröster und Ihr Rächer sein ...“ aus seinem Brief an Guido Lehmann vom 13. Februar 1863 in Aprents ,bearbeitender“ Abschrift ebenso kurzerhand wie eindeutig, und das heißt eindeutig verfälschend 


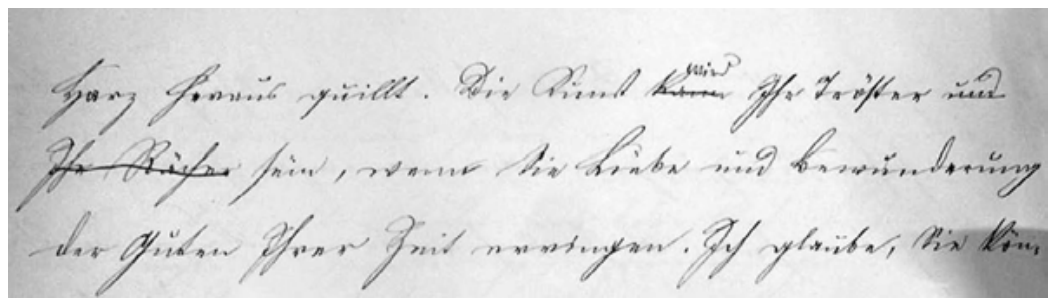

Abb. 1: Johannes Aprent: Abschrift eines Stifter-Briefes mit ,Bearbeitung‘ Aprents.

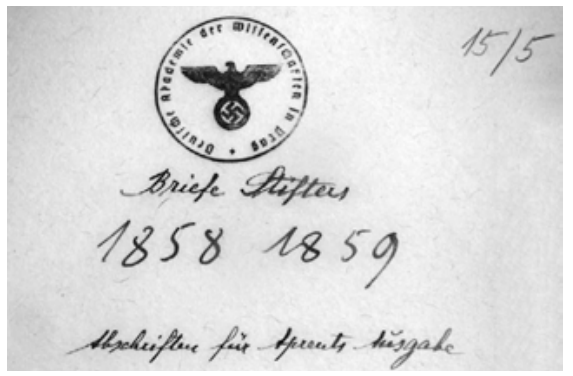

Abb. 2: Stempel aus der Okkupationszeit mit Hakenkreuz auf einer Mappe aus dem Stifter-Archiv, Prag.

zu „Die Kunst wird Ihr Tröster sein ...“ mutiert (Abb. 1). ${ }^{3}$ Glücklicherweise hat Aprent durchweg philologisch exakt gearbeitet, so dass sich in diesen Fällen für die Briefabteilung der $\mathrm{HKG}^{4}$ der ursprüngliche Wortlaut, wo Stifters Hand fehlt, ${ }^{5}$ doch verlässlich rekonstruieren und rekonstituieren lässt.

Wer zumal als deutscher Besucher das Stifter-Archiv konsultiert, wird jedoch mit Befremden und mehr noch mit Unbehagen registrieren, dass die Handschriften dort bis heute in Bögen und Mappen eingeschlagen sind, auf denen nach wie vor und unübersehbar der Stempel der Okkupationszeit mit dem Hakenkreuz in seiner Mitte zu sehen ist (Abb. 2). Ein Unbehagen, das zugleich allerdings auch eine Positionsbestimmung hinsichtlich der beiden Leitbegriffe ,Strategie und Eigendynamik' erlaubt.

3 Die Abschriften Aprents befinden sich unter der Inv.-Nr. StA 15 im Stifter-Archiv. Die Abbildungen 1 und 2 erfolgen mit freundlicher Genehmigung der Abteilung für Handschriften und alte Drucke der Národní Knihovna České Republiky im Prager Klementinum.

4 Die Briefausgabe der HKG - die 11. Abteilung - wird acht Bände umfassen.

5 Die Briefausgabe der PRA übernahm mit dem Hinweis „Original: unbekannt“ (so in den Anmerkungen zur Edition) im zitierten Brief den Wortlaut von Aprents ,Bearbeitung“ (Stifter, PRA 20, 100, 355). 


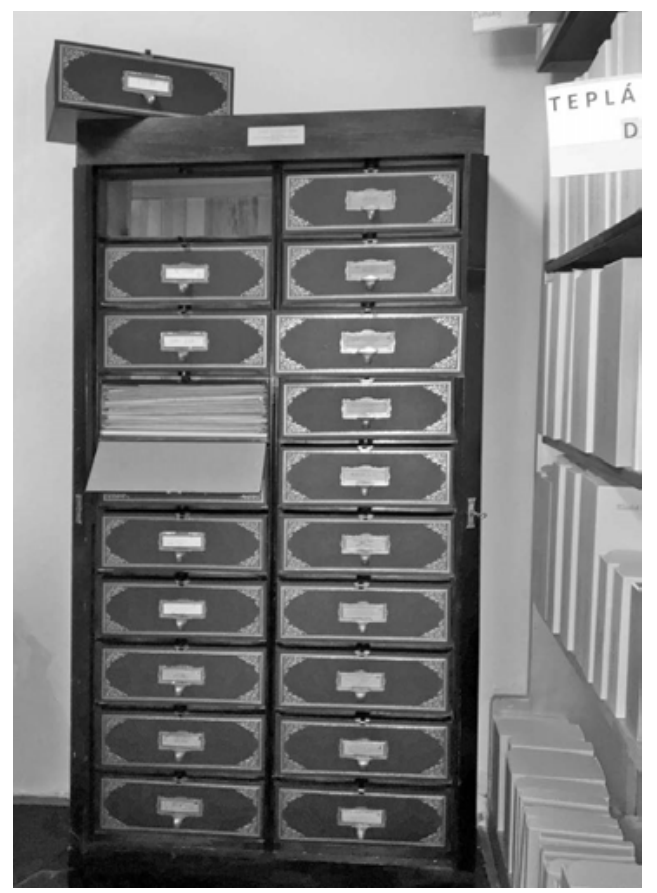

Abb. 3: Prager Stifter-Archiv.

Unter strategischen Gesichtspunkten gehorchte die Entstehung des Prager Stifter-Archivs einer leicht nachvollziehbaren Chrono-Logik. Der Planung einer neuen Werkausgabe musste die möglichst umfassende Sammlung, Sichtung und Katalogisierung der weit verstreuten Materialien folgen, wie sie bis zum Ausbruch des Ersten Weltkriegs dann auch stattfand. Womit freilich die Eigendynamik des Archivs zugleich auch zu einem Stillstand kam. Dies betraf nicht nur mögliche Neuerwerbungen, die zuletzt Mitte der 30er-Jahre erwogen wurden, als das Wiener Antiquariat Gilhofer den in Prag hierfür Verantwortlichen das 380 Blatt umfassende handschriftliche Konvolut des Witiko zum Kauf anbot, welches aus Kostengründen aber nicht erworben werden konnte und sodann in den Besitz der „Fondation Martin Bodmer“ in Genf überging (vgl. Hofman 1984, 111), wo es Alfred Doppler und Wolfgang Wiesmüller in den 80er-Jahren für die in Innsbruck entstandene, 2001 abgeschlossene 5. Abteilung der HKG sichten und auswerten konnten. ${ }^{6}$

6 Die fünf Bände von Lesetext (Stifter, HKG 5.1-5.3; 1984-1986) sowie Apparat und Kommentar (Stifter, HKG 5.4 und 5.5; 1998 und 2001), alle herausgegeben von Alfred Doppler und Wolfgang Wiesmüller, ergänzt und komplettiert eine über das Internet zugängliche Präsentation der 800 
Auch wurde in Prag nie eine grundlegende Neuordnung des Bestandes vorgenommen, was dem in 20 schwarzen Kästen untergebrachten Stifter-Archiv zugleich einen musealen Charakter verleiht (Abb. 3). Dies erlaubt es zwar, mit gewissermaßen archäologischem Blick bis heute die Zusammensetzung der Sammlung in ihren einzelnen Bestandteilen und hier insbesondere den Fundus der diversen, z. T. umfänglichen Schenkungen nachzuvollziehen, eine Reorganisation - wie sie etwa im Falle der an Stifter gerichteten, bislang nach Absendern alphabetisch geordneten Briefe unter chronologischer Reihung sicher sinnvoll gewesen wäre - ist, wie Alois Hofman (1984) selbstkritisch anmerkte, bis heute ein Desiderat geblieben. ${ }^{7}$ Wissenschaftlich aufgewogen wird dies durch die von der Prager Nationalbibliothek vorgenommene partielle Digitalisierung der Bestände, die zwar noch nicht komplett oder allgemein zugänglich sind, der HKG je nach Bedarf aber zur Verfügung gestellt wurden, was deren Publikationsrhythmus wesentlich unterstützt und beschleunigt hat, da die (Vor-)Arbeit am Bildschirm in den meisten Fällen vor Ort nur noch punktuelle Überprüfungen nötig machen. ${ }^{8}$

Rückt damit die seit 1978 im Stuttgarter Kohlhammer-Verlag erscheinende HKG ins Blickfeld, so unterscheidet sich deren Genese geradezu diametral von der eben skizzierten Entstehungsgeschichte der PRA, wenngleich auch sie ein Politikum sui generis bildete, und zwar mit den Antipoden Linz und München, die am 27. November 1964 in Hamburg aufeinandertrafen, um das martialische, wenngleich hier durchaus angebrachte Wort vom ,Kriegsschauplatz' zu vermeiden. ${ }^{9}$ Kurz zuvor nämlich war ein umfängliches Konvolut bis dato unbekannter Handschriften Stifters, das nicht weniger als 14 vollständige Manuskripte des

sogenannten ,abgelegten Blätter zum Witiko in Transkriptionen: Internet Edition Adalbert Stifter - Witiko (Innsbruck 1999-2008). Abrufbar unter: https://www.uibk.ac.at/germanistik/stifter/ witiko/ (25.11.2019).

7 Allerdings hat Hofman (1962, 71-109) diese Chronologie ebenso wie für die von Stifter verfassten Briefe in seiner Übersicht der Sammlungen vorgenommen.

8 Für diese kollegiale Hilfestellung sei an dieser Stelle Kamil Boldan, Miloš Dostál und Petra Hofbauerová herzlich gedankt!

9 Zur besseren Lesbarkeit des Beitrages finden sich die bibliografischen Angaben zu jenen Zeitungsartikeln, aus denen im Folgenden zitiert wird, in narrativer Form im Fließtext; im Literaturverzeichnis werden die Zeitungsbeiträge nicht mehr eigens angeführt. - So prophezeite schon „R. B.“ in den Düsseldorfer Nachrichten vom 30. Oktober 1964, „daß um den Erwerb der Handschriften ,eine wilde Schlacht“ entbrennen“ werde. Von einem „kurzen Duell zwischen der Bayerischen Staatsbibliothek, dem Linzer Stifter-Archiv und anderen“ spricht unter dem Datum des 29. November 1964 „W. H.“ in Die Welt, Nr. 279. „Um Stifter wurde gekämpft“, vermeldeten wortgleich am 7. Dezember 1964 die Düsseldorfer Nachrichten sowie die Westdeutsche Zeitung in ihren Ausgaben Krefeld und Mönchen-Gladbach. Vergleichsweise moderat dagegen Schacherls (1965) Wendung vom ,atemberaubende[n] Konkurrenzkampf“. 
dichterischen Werks von den Studien über die Bunten Steine bis zum Nachsommer enthielt, im wahrsten Sinne des Wortes auf den Markt gekommen, was gleichermaßen „von der Fachwelt wie von der Presse als Sensation empfunden“ (Moisy 2014, 49) wurde. Sie stammten aus dem Besitz des passionierten Sammlers Salman Schocken (1877-1959), dessen Nachfahren diese Bestände dem Hamburger Auktionshaus Dr. Ernst Hauswedell zur Versteigerung anvertraut hatten.

Empfand sich nun - aus naheliegenden Gründen - Linz, und damit als Financier der Staat Österreich als legitimer Anwärter auf diese der Forschung reichstes Neuland eröffnenden Materialien, trat für die Öffentlichkeit möglicherweise unerwartet ${ }^{10}$ mit dem Freistaat Bayern nun auch ein Konkurrent auf den Plan, der es der Münchner Staatsbibliothek schließlich ermöglichte, in einer „erbitterten Steigerungsschlacht“"11 das Tafelsilber des Konvoluts zu dem für damalige Verhältnisse sensationellen Endpreis von 625.000 DM, mithin dem Vierfachen des ausgerufenen Schätzpreises von 156.600 DM, in ihren Besitz zu bringen. Das Pressecho (vgl. exemplarisch Abb. 4 im Anschluss an den Beitrag) war immens, die Verbitterung auf österreichischer Seite ebenfalls, wie sich insbesondere einer offiziellen Presseerklärung des Stifter-Instituts - veröffentlicht in seiner Vierteljahrsschrift 1965 mit der Absicht, „offenkundige Falschmeldungen nicht zur ,historischen Wahrheit“ werden zu lassen“ (Adalbert-Stifter-Institut des Landes Oberösterreich 1965, 142) ${ }^{12}$ - entnehmen ließ, wo auch die logistischen Umstände während der Auktion selbst detailliert nachgezeichnet und als unfair gebrandmarkt wurden, hatte sich der bayerische Vertreter doch nicht wie der Linzer Konkurrent auf den Ehrenplätzen in den beiden ersten Reihen, sondern vielmehr in der Tiefe des Raumes platziert, was auktionsstrategisch freilich immer von Vorteil ist, da man so - fußballterminologisch formuliert - das Spielfeld in seiner ganzen Länge und Breite vor sich liegen hat:

Die ersten zwei Sitzreihen waren für die Hauptinteressenten reserviert. Der Vertreter von Linz waren der Meinung, er würde während der Auktion den Vertreter Münchens kennenlernen. Leider mußte er feststellen, daß sein Konkurrent gar nicht in den vordersten Sitzrei-

10 Wie dessen Presseerklärung zu entnehmen ist, war das Stifter-Institut bereits vorab informiert, „daß die Bayerische Staatsbibliothek die Handschriften unter allen Umständen und mit höchstem finanziellem Einsatz erwerben wollte“ (Adalbert-Stifter-Institut des Landes Oberösterreich 1965, 140).

11 So die Sudetenpost. Offizielles Organ der Sudetendeutschen Landsmannschaft in Österreich am 18. Dezember 1964. Die Überschrift Drei Millionen für Stifter-Handschriften rechnet den Erlös der Auktion - „Über eine halbe Million DM, dazu 15 Prozent Aufgeld ...“ - in österreichische Schillinge um.

12 Als Unterzeichner firmiert „das Adalbert Stifter-Institut des Landes Oberösterreich“, dem als Leiter Aldemar Schiffkorn (1915-1987) vorstand. 
hen Platz genommen hatte. Als die Ausrufungspreise in der Folge im buchstäblichen Sinne des Wortes hinauflizitiert wurden, da zeigte Herr Dr. Hauswedell jeweils über die Köpfe der in den ersten Reihen Sitzenden hinweg, wenn der Vertreter Münchens mitsteigerte. Es bestand also die Absicht, mit dem Vertreter von Linz nicht zusammenzukommen. Das ist die Wahrheit, die niemand bestreiten kann! ${ }^{13}$

Wenig überraschend dominierte den Pressespiegel und hier vor allem die diversen Schlagzeilen vor allem die Magie der großen Zahl, sprich erlösten Summe, ${ }^{14}$ ebenso wenig überraschend mit jeweils denkbar unterschiedlichen nationalen Akzentuierungen. So dürfte das offizielle Linzer Fazit, die angebotenen Handschriften seien letztlich ihren Preis „nicht wert“ gewesen, ${ }^{15}$ verständlicherweise

13 Fritz Hufen erwähnt im Düsseldorfer Handelsblatt vom 30. November 1964 neben dem „Linzer Stifterarchiv“ auch die „Wiener Stadtbibliothek“; das Zitat auf S. 141.

14 Der teure Nachsommer, titelte etwa die Münchner Abendzeitung am 8. Dezember 1964 wie tags darauf auch das Nürnberger 8-Uhr-Blatt; beide Artikel von Karin Thimm. Die Einschätzung der Westdeutschen Zeitung in Mönchengladbach vom 7. Dezember 1964 (Um Stifter wurde gekämpft) mag hingegen zwar für Stifters Wiener Jahre zutreffen, nicht aber für den - freilich von einer zunehmenden Verschuldung bei seinem Budapester Verleger Gustav Heckenast überschatteten - durchaus repräsentativen Lebensstil seiner Linzer Zeit ab 1848: „Stifters Handschriften erbrachten - ein grausiger Hohn auf das armselige Leben des großen Prosaisten - insgesamt 590 000, DM.-“ - Schon vorab hatte Peter Kraft in den Oberösterreichischen Nachrichten am 4. November 1964 von „Ausrufpreisen“ gesprochen, ,die sich bereits in astronomischer Höhe halten“.

15 Mit dieser Formulierung wird am 1. Dezember 1964 gleichlautend im Linzer Volksblatt wie in den Oberösterreichischen Nachrichten Dr. Alois Großschopf (1916-1977) zitiert, der an der Hamburger Auktion als Vertreter des „Stifter-Institutes“ teilgenommen hatte und dieses von 1966 bis 1976 leitete; dass es sich bei Großschopf um einen „gebürtigen Böhmerwäldler (aus DeutschBeneschau)“ handelte, wird von Fritz Huemer-Kreiner im Januarheft 1965 des Böhmerwäldler Heimatbriefs. Monatsschrift für den Heimatkreis Prachtitz eigens erwähnt, was den kulturpolitischen Kontext der Versteigerung nochmals unterstreicht. - Zuvor hatte die in Wien erscheinende Die Presse am 30. November 1964 unter der Überschrift Der verkaufte Stifter das Vorgehen der österreichischen Institutionen hart kritisiert: „Wie rasch wechselten in der Politik Millionen, da es darum ging, eine kleine Koalition zu bevorschussen, und wie armselig verhält man sich zur gleichen Zeit, da es um das geistige Vermächtnis Österreichs geht! Ja, in Rot-Weiß-Rot zu reden, fällt eben leichter, als dafür auch etwas zu tun. Gar, wenn man sich dieses Österreich auch noch was kosten lassen soll! Das Wort: Was du ererbt von deinen Vätern hast, erwirb es, um es zu besitzen, scheint vergessen. So wird man denn auch nichts besitzen.“ Bereits am 16. November 1964 hatte in der Presse Herbert Nedomansky in diesem Zusammenhang die Einrichtung einer Stiftung für Österreich (so der Titel) angemahnt, in den Oberösterreichischen Nachrichten hatte Peter Kraft schon am 4. November 1964 die zuständigen Behörden aufgefordert, diese „Sensation ersten Ranges [...] für Oesterreich und im besonderen für den Hort des Stifter-Instituts zu retten.“ - Für die Bereitstellung österreichischer Pressestimmen sei an dieser Stelle Petra-Maria Dallinger, seit 2004 Leiterin des Adalbert-Stifter-Institutes des Landes Oberösterreich/StifterHaus, sowie deren Mitarbeiterin Margit Auli herzlich gedankt. 
eher cum ira et studio gesprochen worden sein, blieb die österreichische Ausbeute mit zwei Briefen und dem Manuskript der Studien-Mappe demgegenüber doch unbestreitbar bescheiden. ${ }^{16}$

Für unsere Thematik relevant ist in jedem Fall der eminent politische Hintergrund jener Auseinandersetzung, erfolgte Erwerb wie Verbringung der noch unerschlossenen Handschriften nach München doch ganz wesentlich, wenn nicht ursächlich auf Initiative und Druck der dort angesiedelten sudetendeutschen Vertriebenenverbände, die mit dieser Aktion den ,Böhmerwalddichter Adalbert Stifter gewissermaßen exemplarisch wenigstens nach Bayern ,heimholen` wollten, wie die Presseerklärung des Bayerischen Kultusministeriums auch expressis verbis einräumt:

Das Land Bayern hat nach dem letzten Krieg die vertriebenen Deutschen aus dem Sudetenland aufgenommen und ihnen eine neue Heimat gegeben. Bayern leitet daraus das Recht und die Pflicht ab, auch das kulturelle Erbe des Sudentenraums zu übernehmen und zu pflegen. Es hat kein finanzielles Opfer gescheut, den Manuskripten Adalbert Stifters, des Dichter des Böhmerwaldes, in seiner Staatsbibliothek eine neue bleibende Wohnstatt zu breiten. (Zit. n. Herzogenberg 1965, 47) ${ }^{17}$

16 Nur ein kleiner Rest von Adalbert Stifter blieb für Linz, resümierten die Oberösterreichischen Nachrichten in ihrer Überschrift am 1. Dezember 1964.

17 Das Zitat stammt aus dem Munde von „Ministerialrat Karl Böck, jetzt Leiter der Ministerkanzlei des Bayerischen Kultusministeriums“ (Herzogenberg 1965, 47). Die Verlautbarung findet sich auch in einer Presseerklärung der Bayerischen Staatsbibliothek vom Dezember 1964. Der Passus von den ,Rechten und Pflichten findet sich auch in einem für diese Auktion angefertigten Gutachten; darüber hinaus verzeichnet der Erwerbungsakt der Staatsbibliothek - wie ebenso das „Sudetendeutsche Archiv“ - hierzu keine weiteren Unterlagen. - Von Herzogenberg (1921-2012), 1952-1986 Geschäftsführerin, nachfolgend bis 2008 Vorstandsmitglied des Münchner „AdalbertStifter-Vereins“, leitete ihren Beitrag mit den Worten ein: „Dies ist geschehen! Wie Heiligtümer wurden die vom Lande Bayern erworbenen Manuskripte Adalbert Stifters in der Bayerischen Staatsbibliothek ausgestellt und mit diesen Blättern hebt eine neue Epoche der Stifterforschung an, die ihren Schwerpunkt in München haben wird“ (Herzogenberg 1965, 46). Von vergleichbar sakralem Tonfall der Adalbert Stifter in der Staatsbibliothek überschriebene Bericht über die Ausstellung in einem mit „hs“ gekennzeichneten Artikel der Bayerischen Staatszeitung vom 18. Dezember 1964: „In einem stillen Kabinett im ersten Stock des Südflügels der Staatsbibliothek sind die Kostbarkeiten nun bis Weihnachten, aufgeschlagen unter Glas, den Stifter-Freunden zur Betrachtung dargeboten. [...] Es gibt kein Gedränge vor diesen Schaukästen, aber es ist ein stetiges Kommen und Gehen, ein Still-sich-Niederbeugen, Bemühung, die Schriftzüge zu enträtseln, beglückte, halb unterdrückte Ausrufe, wenn ein Wort, ein Satz sich erschloß [...].“ - Für hilfreiche Auskünfte gilt in diesem Zusammenhang der Dank Ingrid Sauer vom Bayerischen Hauptstaatsarchiv sowie Maximilian Schreiber von der Bayerischen Staatsbibliothek München. 
Dass der Anspruch Bayerns, sich „als Treuhänder für die Werke Stifters und darüber hinaus für das Kunsterbe des böhmisch-mährischen Raumes“ zu fühlen, wie ihn das Mitteilungsblatt des Adalbert Stifter Vereins in München im Januar 1965 erhob (vgl. Schacherl 1965, 7-8), gerade in Linz, wo Stifter seit 1848 bis zu seinem Tod nicht nur als Dichter und Maler, sondern ebenso seit 1851 in amtlicher Tätigkeit als k. k. Schulrat wie als Landeskonservator für die Kunstschätze Oberösterreichs und Mitglied des Linzer Kunstvereins vielfältig gewirkt hatte und wo seit den 50er-Jahren des 20. Jahrhunderts das Adalbert-Stifter-Institut sich intensiv dessen wissenschaftlicher Pflege widmete, jedoch als verbale Provokation wirken musste, ist leicht nachvollziehbar, wenngleich München bereits zuvor schon im Besitz wichtiger Manuskripte, etwa der Brigitta, gewesen war (vgl. Landthaler 1967). Auch wenn zudem wichtige Werkausgaben nach dem Zweiten Weltkrieg - genannt seien nur die von Max Stefl herausgegebenen „Urfassungen“ der Stifter'schen Erzählungen (Stefl 1950-1952) ${ }^{18}$ - in Bayern erschienen waren, sind die Replik auf diesen (Alleinvertretungs-)Anspruch wie auch das Schlusswort der Erklärung des Stifter-Instituts von kaum bestreitbarer Stichhaltigkeit:

\begin{abstract}
Mit welchem Recht sich Bayern und München eine „Treuhänderschaft“ selbst zugesprochen haben, bleibt unerfindlich. Hätte nicht Österreich eher ein Anrecht auf die Handschriften gehabt? Im Gymnasium zu Kremsmünster empfing Stifter die Grundlagen seines Wissens und seiner Bildung; in Wien wurde sein Ruhm als Dichter begründet; in Oberösterreich entfaltete er seine segensreiche Tätigkeit als Landesschulinspektor und als Konservator der Zentralkommission zur Erforschung und Erhaltung der Baudenkmäler; in Oberösterreich schrieb er seine großen Werke; in Linz liegt er begraben und vor dem Landhaus zu Linz steht sein schönstes Denkmal. Hat man in München nicht gewußt, daß unser Institut sich die Erforschung von Stifters Leben und Werk schon seit dem Jahre 1950 angelegen sein läßt, daß hier Stifter-Forscher und -Freunde, Lehrer und Schüler aus aller Herren Länder einund ausgehen? [...] Niemand wird abstreiten können, daß aus den Werken Stifters der österreichische Geist in seiner schönsten und reinsten Form spricht, ein Geist, der überall zu Hause sein kann. Es gibt keine Treuhänderschaft über diesen Geist, und man kann dem Dichter nach seinem Tode kein neues Bürgerrecht verleihen. (Adalbert-Stifter-Institut des Landes Oberösterreich 1965, 141-142) ${ }^{19}$
\end{abstract}

Von einem infolge der Umbrüche wie Öffnungen des Epochenjahres 1989 nunmehr leichter möglich gewordenen transnationalen Verständnis Stifters als einem im böhmischen Oberplan, dem heutigen Horní Plana, geborenen österrei-

18 Die HKG ersetzt den Terminus „Urfassungen“ für die Studien (HKG 1.1-1.3) und die Bunten Steine (HKG 2.1) durch die Bezeichnung ,Journalfassungen“.

19 Vgl. zu den hier genannten Stationen Kremsmünster, Wien und Linz den auch Stifters Geburtsort Oberplan einbeziehenden vierteiligen Konferenz-Zyklus über Stifters Welten (2014-2017); veröffentlicht in: Becher und Mayer (2017); Dallinger und Hofer (2018). 
chischen Schriftsteller deutscher Zunge, war man damals noch weit entfernt, wobei rezeptionsgeschichtlich hier der Anteil von Literatur und Forschung an einem sich zumal in den 80er-Jahren grundlegend wandelnden, neuen, nun nicht mehr harmonisierenden oder idyllisierenden Stifter-Bild nicht unerwähnt bleiben darf (vgl. John und Wiesmüller 2018, 374-377).

Durchaus spannend, wenngleich an dieser Stelle nicht weiter zu vertiefen, sind dabei die Frontlinien, wie sie innerhalb der strukturell bis heute föderalistisch verfassten bundesdeutschen Kulturpolitik auch publizistisch auf landespolitischer Ebene verliefen. So mahnten etwa das Düsseldorfer Handelsblatt wie die Westdeutsche Zeitung wortgleich einen doch pfleglicheren Umgang mit Steuergeldern an, wozu freilich angemerkt werden muss, dass der Freistaat Bayern seine Summe aus einem speziellen Fond staatlicher Sondermittel und nicht etwa laufenden Budgets zur Verfügung gestellt hatte. ${ }^{20}$ Dazu mag beigetragen haben, dass sich auch das in Frankfurt angesiedelte „Freie Deutsche Hochstift“ zumindest auf Teilerwerbe Hoffnungen gemacht hatte, eine „Preistreiberei“21 befürchtend aus dem Wettbewerb aber bereits vor dem Hamburger Showdown ausgestiegen war. ${ }^{22}$ Am polemischsten fiel hier Fritz Hufens Verdikt aus, wonach mit dem Münchner Zuschlag die „Sicherungen des gesunden Menschenverstandes und

20 Hierzu Karin Thimm (Anm. 15) im 8-Uhr-Blatt Nürnberg in ihrem Bericht über die Pressekonferenz in der Münchner Staatsbibliothek: „Die halbe Million Mark aber, die man jetzt für Stifters Manuskripte ausgab, fallen mit keinem Pfennig dem Etat zur Last. Kultusministerium und Finanzministerium haben sie aus dem Grundstückvermögen des Staates losgeeist, als eine Wertanlage sozusagen, zur Vermehrung des Staatsbesitzes.“

21 So Christian Otto Frenzel (Eine halbe Million für Stifters Manuskripte) in der Frankfurter Allgemeinen Zeitung vom 30. November 1964.

22 Allerdings differiert in der Presse hierzu die Berichterstattung. Die Hinweise auf das „Hochstift“ als Interessenten im Münchner Merkur vom 2. Dezember (Immer mehr Stifter in München) und der Sudetenpost vom 18. Dezember 1964. Die Passauer Neue Presse vom 17. Dezember 1964 hingegen meldet unter dem Titel Stifter ist sein Geld wert, dass beide, das „Stifter-Museum in Linz und das Freie Deutsche Hochstift in Frankfurt [...] lebhaft mitgeboten hatten“. Dem steht die Aussage von Johanna von Herzogenberg $(1965,46)$ entgegen, wonach der Entschluss zur Abstinenz bereits im Vorfeld getroffen wurde: „Es verhandelten also die öffentlichen HandschriftenSammlungen miteinander - in Deutschland stehen an erster Stelle das Schiller-Nationalmuseum in Marbach und das Freie Deutsche Hochstift in Frankfurt, welche beide zurücktraten, als sie die guten Gründe der Bayerischen Staatsbibliothek hörten.“ - In gleichem Sinne zuvor schon Erhard Göpel (Bayern ehrt Adalbert Stifter) in einem umfänglichen Bericht der Süddeutschen Zeitung vom 12./13. Dezember 1964: „Andere deutsche Stellen, wie das Freie Deutsche Hochstift in Frankfurt, mit denen auf der Ebene der Kultusministerkonferenz eine Verständigung gesucht worden war, hatten darauf verzichtet, die von der ,Stiftung Volkswagenwerk ‘ zur Verfügung gestellten Mittel gegen München auszuspielen, das von Anfang an mit Entschiedenheit seine Erwerbsabsicht bekundet hatte.“ 
der Marktkenntnis“ durchgebrannt seien, „die eigentlich die öffentlichen Bibliotheken vor törichten Handlungen bewahren sollten“. ${ }^{23}$ Bemerkenswert jedenfalls, welch breites Echo ein kulturelles, eigentlich ja (fach-)wissenschaftliches Sujet seinerzeit hervorzurufen vermochte. ${ }^{24}$ Wie es in unserem Zusammenhang ebenfalls festzuhalten gilt, dass damit München - so die Formel einer auffällig einmütigen Sprachregelung im Feuilleton - avant la lettre zum (künftigen), Zentrum der Stifter-Forschung' avancierte. ${ }^{25}$

Wurde aus naheliegenden Rechtfertigungsgründen dieser Erwerb bzw. diese Investition von Münchner Seite deshalb von Beginn an mit der Notwendigkeit verknüpft, diesen Bestand nunmehr rasch wissenschaftlich $\mathrm{zu}$ erschließen, wofür der Münchner Ordinarius Hermann Kunisch (1901-1991) Zuständigkeit und Verantwortung übernahm, ${ }^{26}$ bedeutet dies unter strategischen Gesichtspunkten, dass hier der Erwerb einer Sammlung aller weiteren Planung voranging, die Handschriften also bereits vor ihrer, ordentlichen‘ Archivierung eine Eigendynamik entfalteten, die dann 1968 zu ersten konkreten Resultaten führte, welche vor dem Hintergrund der eben skizzierten internationalen Verwerfungen und Verstimmungen alles andere als naheliegend oder gar selbstverständlich waren.

1968 nämlich fand anlässlich der 100. Wiederkehr von Stifters Todesjahr im österreichischen Bad Hall ein international besetztes Symposion statt, auf dem sich eine Sektion ausschließlich den ersten Schritten zur Planung einer neuen, das nunmehr zur Verfügung stehenden Archivmaterial auswertenden historisch-

23 So im Düsseldorfer Handelsblatt vom 30. November 1964 unter der Überschrift Teure Triumphe der Bibliotheken. Auch das Linzer Volksblatt vom 1. Dezember 1964 kritisiert die erzielten „Phantasiepreise“.

24 Das Archiv der Münchner Arbeitsstelle bei der „Bayerischen Akademie der Wissenschaften“ - Sitz der Redaktion der HKG - dokumentiert zwischen dem 25. September 1964 und Februar 1965 insgesamt 83 z. T. umfängliche Presseberichte. Waren vor dem Versteigerungstermin sieben Artikel erschienen, liegt der Schwerpunkt der Berichterstattung dann im Zeitraum zwischen dem 30. November und dem 14. Dezember 1964. Am 9. Dezember etwa wurde in nicht weniger als 24 Presseorganen über das Ergebnis der Auktion berichtet. - Im Anschluss an die Erklärung des Adalbert-Stifter-Institutes (1965) veröffentlicht das Institut eine von Max Stefl (1965, 143-144) zusammengestellte, auch als Sonderdruck publizierte tabellarische Publikationsliste: Im Spiegel der Presse.

25 Gerade am 9. Dezember bildet diese Wendung unisono die Überschrift zahlreicher Artikel, etwa im Düsseldorfer Handelsblatt (München als Stifterzentrum), der Mittelbayerischen Zeitung Regensburg (Forschungszentrum für Stifters Schaffen) oder den Salzburger Nachrichten (München wird Zentrum für Stifter) $\mathrm{u}$. ö.

26 Bereits am 8. Dezember 1964 heißt es im Beitrag Der teure Nachsommer der Münchner Abendzeitung hierzu: „So hat Professor Dr. Hermann Kunisch von der Münchner Universität bereits sein und seiner Studenten lebhaftes Interesse an vergleichenden Studien anhand der kostbaren Werke kundgetan.“ 
kritischen Gesamtausgabe widmete. Bemerkenswert bleibt, obwohl die Rivalitäten und Kränkungen der Hamburger Auktion zwischen den Zeilen durchaus noch nachhallten, dabei die Einigkeit, dieses Projekt überregional, heißt: international „unter dem Patronat je einer Institution Deutschlands, Österreichs und der Tschechoslowakei“ (Vancsa 1969, 24)27 in die Wege zu leiten, so im 1969 in der Vierteljahreschrift des Adalbert-Stifter-Institutes veröffentlichten Protokoll jener Tagung. 1973 dann waren, worüber Hermann Kunisch und für den österreichischen Part Alfred Doppler (`1921) wiederum in der Linzer Institutszeitschrift informierten, Die Vorbereitungen für die historisch-kritische Stifter-Ausgabe (so der Titel des Beitrages; Kunisch und Doppler 1973) nochmals konkreter geworden, indem etwa die Einrichtung einer eigenen Arbeitsstelle, angesiedelt bei der Bayerischen Staatsbibliothek und besetzt mit einem hauptamtlichen wissenschaftlichen Mitarbeiter, beschlossen wurde. Der weitere Fortgang in Kürze: 1978 erschienen unter der Hauptherausgeberschaft von Alfred Doppler und Wolfgang Frühwald (1935-2019) die ersten Bände, 1986 ging die Arbeitsstelle in die Obhut der Bayrischen Akademie der Wissenschaften über; im Jahr 2000 folgte Hartmut Laufhütte (*1937) Frühwald als deutscher Hauptherausgeber nach.

Anfänglich gefördert durch die „Deutsche Forschungsgemeinschaft“ (DFG) und die „Thyssen-Stiftung“, seit 2006 durch den Freistaat Bayern sowie durch jährliche Zuschüsse des Landes Oberösterreich wie der Österreichischen Akademie der Wissenschaften, ist die HKG seit ihrem Bestehen nicht nur ein reibungslos funktionierendes österreichisch-deutsches Gemeinschaftsunternehmen und „darf [...] mit Fug bundesweit als eines der erfolgreichsten Editionsprojekte der letzten Jahrzehnte überhaupt gelten“; ${ }^{28}$ sie konnte bis zum Jahr 2018 mittlerweile 39 Bände vorlegen und dabei fünf der insgesamt elf Abteilungen abschließen. Die enge Verzahnung über nationale - freilich kaum spürbare - Grenzen hinweg dokumentiert nicht nur die Zusammensetzung der editorischen Arbeitsgemeinschaft aus momentan sechs deutschen und zwölf österreichischen Kolleginnen und Kollegen, sondern auch die Mitgliedschaft fast aller dieser Bandherausgeberinnen und -herausgeber im Adalbert Stifter-Institut des Landes Oberösterreich.

Wenn aus der apostrophierten Konkurrenz also längst eine nachweislich fruchtbare, angesichts der Vorgeschichte jedoch keineswegs selbstverständliche Kooperation geworden ist, betrifft dies mittlerweile gleichermaßen auch das Verhältnis zwischen den Archiven in München und Linz, wenn sich etwa beide

27 Dort das von Helmut Bergner, dem späteren ersten Redaktor der HKG, verfasste „Protokoll“ des Arbeitskreises I („Probleme einer neuen Gesamtausgabe der Werke, Schriften und Briefe Adalbert Stifters“).

28 So im Evaluierungsgutachten des Projekts aus dem Jahre 2011. 
Häuser schon seit längerem bei Auktionen im Vorfeld strategisch abstimmen, um nicht auf Kosten ihrer Budgets gegeneinander zu bieten - und so im Übrigen jenen Forderungen genügen, wie sie nach der Versteigerung vom November 1964 im Feuilleton nachdrücklich erhoben wurden. ${ }^{29}$ Wechselseitige Auftragserteilungen, die auch den verantwortlichen Redaktor der HKG einbeziehen, sind inzwischen keineswegs die Ausnahme. Zu berücksichtigen ist dabei, dass mit größeren ,Entdeckungen' bis dato unbekannten Materials im Falle Stifters kaum mehr zu rechnen ist und der Erwerb der mehrseitigen, handschriftlich von Stifter für den Privatunterricht im Hause Metternich verfassten Psichologie der Tiere ${ }^{30}$ hier eher die Ausnahme bleiben dürfte, die - nach Begutachtung durch Experten der HKG im Frühjahr 2011 und nachfolgenden Umwegen über verschiedene Anbieter und Reduzierung auf einen zwar immer noch stolzen, aber eher angemessenen Preis - schließlich auf der Frühjahrsauktion des Hauses Stargardt in Berlin im März 2014 vom Stifter-Institut erworben und somit an den Ort verbracht werden konnte, wohin sie auch gehört.

So ist es auch das Stifter-Institut in Linz, das in den letzten Jahren, was Neuerwerb und Komplettierung anbelangt, archivalisch zweifellos die nachhaltigste Dynamik entfaltet hat, was zuletzt auch die Übernahme wichtiger handschriftlicher wie archivalischer Bestände des im Dezember 2018 aufgelösten Wiener „Stifter-Vereins“ verdeutlicht, die dann im Laufe des Jahres 2019 nach Linz übersiedelten. Dessen zentrale Rolle als Erinnerungsort hatte schon Alois Hofman 1984 betont, als er das Prager Stifter-Archiv ausdrücklich als „Teil des Stifter-Hauses an der Linzer Donau-Lände“ (Hofman 1984, 113) bezeichnete: eine doch bemerkenswerte Feststellung, die - immerhin fünf Jahre vor dem Fall des Eisernen Vorhangs - im Kontext von ,Archiv und Aura' gewissermaßen eine Logik der Topografie und Geografie postuliert. Auch die in Linz liegenden Bestände an Stifter-Handschriften sind im Übrigen der HKG auf digitale Weise zugänglich.

Womit - um in der oberösterreichischen Landeshauptstadt zu bleiben - der Standort Linz abschließend noch um ein weiteres wichtiges Depositum erweitert werden soll, liegen im dortigen Oberösterreichischen Landesarchiv in einer separaten Abteilung doch die sogenannten ,Stifter-Akten', die seine Tätigkeit als k. k. Schulrat zwischen 1851 und 1865 dokumentieren und die Textbasis der 10. Abtei-

29 Wenigstens „ein Minimum an Zusammenarbeit“ hatte mit polemischer Schärfe Fritz Hufen am 30. November 1964 im Düsseldorfer Handelsblatt eingefordert; ebenso, wenngleich moderater, Johanna von Herzogenberg mit ihrem Plädoyer, „verantwortlich vorzugehen und unter den ernsten Interessenten eine Absprache so zu treffen, daß sich nicht verwandte Institutionen gegenseitig hochsteigern“ (Herzogenberg 1965, 46).

30 Stifter, HKG 8.2, 13-20. 
lung der HKG bilden, welche die „Amtlichen Schriften zu Schule und Universität“ in sechs Bänden auf rund 2.600 Seiten präsentiert und seit dem Frühjahr 2018 nunmehr abgeschlossen vorliegt. Wenn den drei Textbänden (Stifter, HKG 10.110.3) mit ihren mehr als 600 Dokumenten gleichberechtigt drei Apparat- und Kommentarbände (Stifter, HKG 10.4-10.6) zur Seite stehen, ist auch dies einer den Akten inhärenten Dynamik geschuldet, wie sie der Passauer Bandherausgeber Walter Seifert erstmals in dieser Form freigelegt hat. Während sich Kurt Vancsa (1955) und Kurt Gerhard Fischer (1961) in ihren vorangegangenen Sammlungen in erster Linie auf den Wortlaut von Stifters Gutachten, Eingaben, Memoranden und Inspektionsberichte konzentrierten und nur knapp kommentierten, bezieht Seiferts Edition erstmals den gesamten verfügbaren Aktenbestand der jeweiligen Vorgänge mit ein und dokumentiert so deren Verlauf im Kräfte- und Interessenparallelogramm zwischen Statthalterei, bischöflichem Konsistorium, regionalen Institutionen wie übergeordneten Wiener Behörden von ihrem Anlass über diverse Amtsgänge bis zu deren schließlichem Entscheid en détail, also nicht summarisch resümierend, sondern wo immer geboten mit wörtlichen Belegen. Möglich war dies nur, weil das Landesarchiv hier auf vorbildliche Weise nicht nur die Digitalisate der ,Stifter-Akten', sondern auch deren amtliches Umfeld bereitwillig zur Verfügung gestellt hat, alles in allem rund 30.000 Blatt oberösterreichischer Amts-, Schul- und Sozialgeschichte, die es hierzu freilich penibel zu durchforsten galt. ${ }^{31}$ Was vor nicht allzu langer Zeit noch vor Ort zu leisten gewesen wäre und ohne Frage ein Vielfaches an Zeit erfordert hätte, konnte - was den Publikationsturnus betrifft - so nun innerhalb des angesichts der schieren Masse des Materials doch denkbar kurzen Zeitraums von gerade einmal einer Dekade erfolgen - eine Form der Dynamisierung von Forschung auch dies, die zugleich den Abschluss des Langzeitprojekts der HKG in greifbare Nähe rückt und so der vordringlichsten wie vornehmsten Aufgabe eines jeden Archivs genügt: nämlich seine Bestände in den wissenschaftlichen wie öffentlichen Diskurs einzuspeisen und sie wie jedes historische Dokument so - und nur so - am Leben zu erhalten.

31 Der auf dem Symposium bereits mündlich abgestattete Dank an die Leiterin des Oberösterreichischen Landesarchivs, Cornelia Sulzbacher, wie deren Vorgänger Gerhart Marckhgott, sei hier gerne wiederholt. 


\section{Rekordumsätze auf der Hauswedell-Auktion Hamburg}

$\mathrm{O} / 12$ Stiftermanuskripte für 590000 DM versteigert - Die öffentliche Hand sollte maßhalten

Der Besucherandrang war an allen Tagen -Pinselzeichnungen blieben gefragt. Die der Versteigerung gros, am dritten Tag, der $\begin{aligned} & \text { Bilder waren der dritte Hohepunkt der C C } \\ & \text { ausschleBlich der Kunstauktion }\end{aligned}$ gab es drangvolle Fulle. Das deutsche und
(Schâtzpreis 120000 ) DM zugeschlagen.

\section{0/42 Stifter iff fein B̧elo wert}

Ba

Dine halbe Malion Mark aut die Hand, Fragmente, meist nur Binzelbialter, nachige

und so mandier unter uns Stantsolirgern hatte wiesen werden. Die historisch-kritische Aus-

Ahnung davon hat, das Adalbert Stifter (1806 meist aut die felierhafter

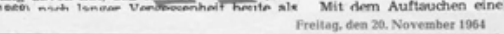

\section{Mark für Adalbert Stifter}

Drei Millionen für StifterHandschriften eine halbe Million DM, a den dazu 15 Prozent Aufgeld, brachten Original, = und handschriften von Adalbert Stifter, darunter mslos die Drudkvorlagen fur die Bunten Steine" at und und den "Nachsommer", bei der 134. Auktion 1 Andes Hauses Hauswedell in Hamburz, Aus einer erbitterten Steigerunsschlacht mit dem StifterArchiv in Linz und dem Freien Deutschen
Für 144500 Mark Stifter

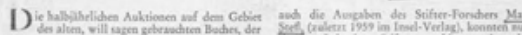

\section{Immer mehr Stifter in München}

\section{D $1 / 2$ Hohe Auktionsergebnisse in Hamburg} wedell in Hamburs die Sensationen, die | Ernst Hauswedell, Hamburg, waren die Die bej
von 19

$$
\begin{gathered}
\text { Kölner Stadtanzeiger, Köln } \\
\text { vom L4. Dez.1984 }
\end{gathered}
$$
W if bereits kurz gemeldet, erbrachten $Y$ rungen des Kunstauktionshauses Dr. wir an dieser Stelle vorausgesagt hatten.
Die bel

Zu den Neuerwerbungen der Bayerischen Staatsbibliothek

Wenn mir einmal das Geschick sönnen im Adam-Kraft-Veriag, Auch Protess Die kostbaren Handschriften des sollte, meinen dauernden Wohnsitz in Kunisch, der Ordinarius fur neuere deu
Manchen nehmen zu können, wirde fich sche Philologie an der Universituit Mü dich zuweilen mit meiner Gegenwart pla- chen, beschilftigt sich intensiv mit St Sehnsucht Adalbert Stifters in einem Zeitschriften erschien 1950 sein Bu Schulmeisters Adalbert Stifter 0 lìn Oberösterreich war nicht konkurrenzfăhig

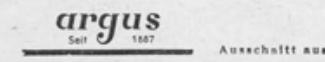

Bayreuther Tageblatt, Bayreuth

$$
\text { vom 11. Dez.1965 }
$$

\section{Forschungszentrum für Stifter}

\section{Muhchén besitzt großen Teil der Manuskripte des Dichter}

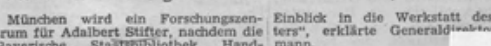

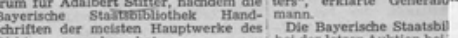
Der Gerworben hat. taatsbibllothek, Dr. Gustav Hof- 625000 DM eriteigert. nann, betonte, die neuerworbenen um die Werke Bunte

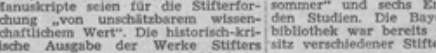

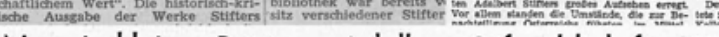

Nur ein kleiner Rest von Adalbert Stifter blieb für Linz

Die grofye Hamburger Auktion von Adalbert Sfiffer-Manuskriplen sah das Ausland finanziell im Vorteil

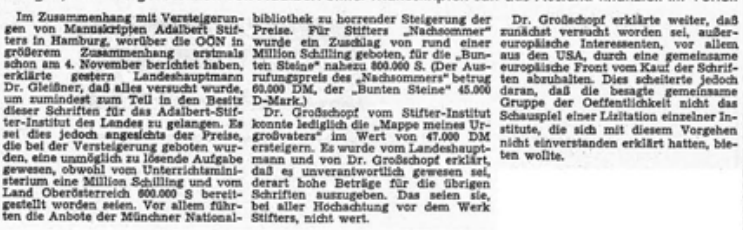

$\mathrm{d} / \mathrm{H}_{2}$ München als Stifterzentrum

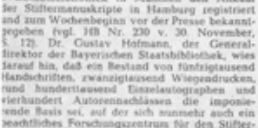

Abb. 4: Collage von Presseberichten zur Versteigerung von Stifter-Handschriften 1964. 


\section{Literaturverzeichnis}

Adalbert-Stifter-Institut des Landes Oberösterreich. „Die Versteigerung von Stifter-

Handschriften in Hamburg“. Vierteljahresschrift des Adalbert-Stifter-Institutes des Landes

Oberösterreich 14 (1965), F. 3/4: 140-142.

Aprent, Johannes (Hg.). Briefe von Adalbert Stifter. 3 Bde. Pest: Gustav Heckenast, 1869.

Becher, Peter, und Franziska Mayer (Hg.). Stifters Welten 1: Oberplan; Stifters Welten 2:

Kremsmünster. Linz: Adalbert-Stifter-Institut, 2017 (= Jahrbuch des Adalbert-StifterInstitutes Bd. 24).

Bergner, Helmut. „Band 25 der Prag-Reichenberger Stifter-Ausgabe. Kritische Anmerkungen zu einer Nachlaßpublikation und zu einer Edition des ,Julius““. Vierteljahresschrift des Adalbert-Stifter-Institutes des Landes Oberösterreich 31 (1982), F. 1/2: 33-72.

Dallinger, Petra-Maria, und Georg Hofer (Hg.). Stifters Welten 3: Wien; Stifters Welten 4: Linz. Linz: Adalbert-Stifter-Institut, 2018 (= Jahrbuch des Adalbert-Stifter-Institutes Bd. 25).

Doppler, Alfred: „Adalbert Stifters Briefe als Dokumente der Selbstdarstellung“. Stifter und Stifterforschung im 21. Jahrhundert. Biographie - Wissenschaft - Poetik. Hg. Alfred Doppler, Johannes John, Johann Lachinger und Hartmut Laufhütte. Tübingen: Niemeyer, 2007. 1-12.

Fischer, Kurt Gerhard. Documenta Paedagogica Austriaca. Adalbert Stifter. Zusammengestellt und mit einer Einleitung versehen von K. G. F. 2 Bde. Linz: Landesverlag, 1961.

Herzogenberg, Johanna von. „Bayern erwirbt Stifter-Manuskript“. Sudentenland. Vierteljahresschrift für Kunst, Literatur, Wissenschaft und Volkstum 1 (1965): 46-49.

Hettche, Walter, und Johannes John. „Editionsgeschichte“. Stifter-Handbuch. Leben - WerkWirkung. Hg. Christian Begemann und Davide Giuriato. Stuttgart: Metzler, 2017. 365-368.

Hofman, Alois: „Die Sammlungen des Prager Adalbert Stifter-Archivs“. Vierteljahresschrift des Adalbert-Stifter-Institutes des Landes Oberösterreich 11 (1962), F. 3/4.

Hofman, Alois. „Bericht über das Prager Adalbert-Stifter-Archiv“. Vierteljahresschrift des Adalbert-Stifter-Institutes des Landes Oberösterreich 33 (1984), F. 3/4: 109-113.

Höhne, Steffen. „Germanistik in den Böhmischen Ländern“. Handbuch der deutschen Literatur Prags und der Böhmischen Länder. Hg. Peter Becher, Steffen Höhne, Jörg Krappmann und Manfred Weinberg. Stuttgart: Metzler, 2017. 17-23.

Hoskovec, Tomáš: „Klub a Kroužek. Úvahy o dynamice české filologie“. Moderní filologie na prahu třetího tisíciletí. Vybrané příspěvky z konference $k 100$. Výročí založení KMF (FFUK Praha 17. 2. 2010). Praha: Kruh moderních filologu, 2010. 26-40.

Jammers, Antonius, Dietger Pforte und Winfried Sühlo (Hg.). Die Besondere Bibliothek oder: Die Faszination von Büchersammlungen. München: Saur, 2002.

John, Johannes, und Wolfgang Wiesmüller. „Rezeption und Wirkung“. Stifter-Handbuch. Leben Werk - Wirkung. Hg. Christian Begemann und Davide Giuriato. Stuttgart: Metzler, 2017. 368-378.

Kunisch, Hermann, und Alfred Doppler. „Die Vorbereitung für die historisch-kritische StifterAusgabe“. Vierteljahresschrift des Adalbert-Stifter-Institutes des Landes Oberösterreich 22 (1973), F. 3/4: 89-92.

Landthaler, Wolfgang. „Münchener Stifter-Handschriften“. Literaturwissenschaftliches Jahrbuch. Hg. Hermann Kunisch (Im Auftrage der Görres-Gesellschaft). Neue Folge. Bd. 8. Berlin: Duncker \& Humblot, 1967. 119-155. 
Moisy, Sigrid von. „Salman Schocken und die Adalbert Stifter-Sammlung der Bayerischen Staatsbibliothek“. BibliotheksMagazin. Mitteilungen aus den Staatsbibliotheken in Berlin und München 3 (2014): 48-52.

Riener, Karoline. „August Sauer und Adalbert Stifter“. August Sauer (1855-1926). Ein Intellektueller in Prag zwischen Kultur- und Wissenschaftspolitik. Hg. Steffen Höhne. Köln, Weimar, Wien: Böhlau, 2011. 283-307.

Rossner, Christiane. „Räume der Erkenntnis“. Monumente. Magazin für Denkmalkultur, April 2016: 8-15.

Schacherl, Lilly. „Eine halbe Million für Stifters Manuskripte. Überraschend aufgetauchte Handschriften von der Münchner Staatsbibliothek erworben“. Mitteilungsblatt des Adalbert Stifter Vereins 1/XIII (Januar 1965): 7-8.

Schley, Fridolin: „Von Büchern, Bäuchen und Bekloppten. Ein Erfahrungsbericht aus der Staatsbibliothek“. Süddeutsche Zeitung, 2.1.2008.

Stefl, Max (Hg.). Adalbert Stifter. Erzählungen in der Urfassung. 3 Bde. Augsburg: Adam Kraft, 1950-1952.

Stefl, Max. „Im Spiegel der Presse“. Vierteljahresschrift des Adalbert-Stifter-Institutes des Landes Oberösterreich 14 (1965), F. 3/4: 143-144.

Stifter, Adalbert. Sämmtliche Werke (= Prag-Reichenberger-Ausgabe). Hg. August Sauer. Calve u. a.: Prag u. a., 1901ff. [Textbelege aus dieser Ausgabe erfolgen unter der Sigle PRA mit Band, Seitenzahl.]

Stifter, Adalbert. Werke und Briefe. Historisch-Kritische Gesamtausgabe. Hg. Alfred Doppler und Wolfgang Frühwald [seit 2000 Alfred Doppler und Hartmut Laufhütte]. Stuttgart u. a.: Kohlhammer, 1987ff. [Textbelege aus dieser Ausgabe erfolgen unter der Sigle HKG mit Band und Seitenzahl.]

Stüben, Jens. „Stifter-Editionen“. Editionen zu deutschsprachigen Autoren als Spiegel der Editionsgeschichte. Hg. Rüdiger Nutt-Kofoth und Bodo Plachta. Tübingen: Niemeyer, 2005. 403-431.

Tvrdík, Milan: „Die tschechische Germanistik nach 1945“. Germanistik in Mittel- und Osteuropa 1945-1992. Hg. Christoph König. Berlin, New York: de Gruyter, 1995. 248-255.

Vancsa, Kurt (Hg.). Die Schulakten Adalbert Stifters. Mit einem Anhang (Personalakten, Organisations-Entwurf der Linzer Realschule). Graz, Wien: Stiasny, 1955. 
